\title{
Effects of SCH 23390 on motivational aspects of deprivation-induced feeding
}

\author{
ULRIKE KOECHLING, LOIS M. COLLE, and ROY A. WISE \\ Concordia University, Montreal, Quebec, Canada
}

\begin{abstract}
The selective D-1 dopamine antagonist SCH 23390 caused a rapid but progressive increase in mean latency to initiate eating and an almost immediate decrease in mean time to complete the eating of five 45-mg food pellets within 36-sec meal segments in food-deprived rats. While mean scores were slowed, normal latencies and speeds were still noted in a significant portion of trials. The drug did not decrease the absolute limits of peak performance; rather, it increased the variance of both response measures. While the effects of SCH 23390 developed more quickly than those reported for the preferential D-2 blocker pimozide, the effects were qualitatively similar and consistent with emerging views of interaction between D-1 and D-2 receptor mechanisms and of the involvement of dopamine in motivational aspects of behavior.
\end{abstract}

Neuroleptic drugs attenuate feeding (Blundell \& Latham, 1978, 1982; Heffner, Zigmond, \& Stricker, 1977; Rolls et al., 1974; Wise \& Colle, 1984; Wise \& Raptis, 1986) and food-rewarded instrumental behavior (Ahlenius, 1979; Beninger et al., 1987; Mason, Beninger, Fibiger, \& Phillips, 1980; Wise, Spindler, de Wit, \& Gerber, 1978; Wise, Spindler, \& Legault, 1978) in laboratory animals. The pattern of response decrement both within (Mason et al., 1980; Sanger, 1986; Wise, Spindler, de Wit, \& Gerber, 1978; Wise, Spindler, \& Legault, 1978) and across (Beninger et al., 1987; Mason et al., 1980; Wise, Spindler, de Wit, \& Gerber, 1978) drug tests raises the possibility (Wise, 1982) that these drugs attenuate the rewarding impact of food; this possibility is more forcefully suggested by studies in which the effects of neuroleptics are reflected in enhanced performance during drug-free tests that are given following experience under the neuroleptic (Ettenberg \& Camp, 1986a, 1986b; Spyraki, Fibiger, \& Phillips, 1982). Neuroleptics are also thought to attenuate the rewarding effects of psychomotor stimulants (de Wit \& Wise, 1977; Risner \& Jones, 1976, 1980; Yokel \& Wise, 1975, 1976) and electrical stimulation of the brain (Beninger \& Freedman, 1982; Fenton \& Liebman, 1982; Fouriezos, Hansson, \& Wise, 1978; Fouriezos \& Wise, 1976; Franklin \& McCoy, 1979; Gallistel, Boytim, Gomita, \& Klebanoff, 1982; Liebman, 1983; Zarevics \& Setler, 1979).

In most cases, the reward-attenuating effects of various neuroleptics seem well correlated with the affinity of these drugs for the D-2 rather than the D-1 dopamine receptor (Gallistel \& Davis, 1983; Lynch \& Wise, 1985). An exception to this generalization is the highly selective (Hyttel, 1983) D-1 antagonist SCH 23390; this agent causes patterns of response decrement in food-rewarded (Beninger et al., 1987; Nakajima, 1986) and brain-

Address correspondence to R. Wise, Department of Psychology, Concordia University, 1455 de Maisonneuve Blvd. West, Montreal, PQ H3G 1M8, Canada. stimulation-rewarded (Nakajima \& McKenzie, 1986) animals that are similar to those caused by the preferential D-2 antagonists haloperidol and pimozide. Moreover, SCH 23390, like preferential D-2 antagonists, suppresses sucrose intake in food-deprived rats (Schneider, Gibbs, \& Smith, 1986a, 1986b).

Since parkinsonian patients report an inability to initiate voluntary movements, and since neuroleptic drugs have parkinsonian side effects (Barbeau, 1974; Hornykiewicz, 1975), it is important to assess the motoric capability of neuroleptic-treated animals. Only if the performance capacity of the animals appears to be normal or near normal should further consideration be given to the possibility that dopamine-blocking drugs impair the motivation of the animal by reducing the rewarding feedback from food. The "typical" neuroleptic pimozide is known to alter the motivation rather than the absolute ability to feed normally. This is reflected in two findings. First, pimozide alters the capacity of food to sustain feeding at a normal rate before it alters the capacity of food to initiate feeding (Wise \& Colle, 1984; Wise \& Raptis, 1986). Second, it decreases mean performance (in terms of both speed and latency measures) on some trials while leaving it normal on others; thus it alters mean performance by increasing the variability of scores-increasing the number of trials in which other behaviors intervene-rather than by adding a constant to each score and thus shifting the entire distribution of scores to slower values (Wise \& Colle, 1984; Wise \& Raptis, 1986). Shifts in mean score without an increase in score variability are to be expected if the limits of the animal's performance are somehow reduced; shifts in mean score that result from increased variability of performance offer an operational definition for the notion of a motivational deficit (Wise, 1987).

The purpose of the present study was to examine the effects of SCH 23390 in the feeding paradigm that has been used to characterize the motivational deficits associated with the preferential D-2 antagonist pimozide. 
The paradigm involves the resolution of a meal into several discrete meal segments, in which the speed of eating and the latency to initiate eating are separately measured. "Best-scores analysis" is then used to determine whether the drug causes deficits of a motivational (increase in mean and variance) or a motor (increase in mean with no increase in variance) classification.

\section{METHOD}

Twelve adult male Long-Evans rats, housed individually and maintained with restricted access to food, were used. The animals were trained to take $20 \%$ of their daily food ration in test boxes where 18 meal segments, each consisting of five 45-mg Noyes food pellets, were offered for $36 \mathrm{sec}$ with 36-sec intervals between offerings. Immediately following testing, each animal was returned to its home cage and given $20 \mathrm{~g}$ of normal laboratory chow. The animals usually consumed this amount of food within 4-6 h, and were thus, in effect, maintained on an 18-h food-deprivation schedule.

The meal segments were delivered automatically. Lab-made feeding platters protruded $3.5 \mathrm{~cm}$ into $25 \times 25 \mathrm{~cm}$ test boxes. Thirtysix food cups $(1.3 \mathrm{~cm}$ diameter $\times 0.8 \mathrm{~cm}$ deep) were drilled into the 25 -cm-diam platters; one of the 36 cups was exposed through a mask at any given time. The platters were indexed one position every $36 \mathrm{sec}$; rubber drive wheels on continuously running motors were pulled against the circumference of the platter by a solenoid when triggered by a timer. The solenoid circuit was broken by a microswitch when a new food cup reached the correct position. The solenoid noise was clearly audible but not loud; it could just be heard over normal conversation. The aluminum food platters were vacuumed, scraped clean, or washed with distilled water; minerals in tap water can interact with the aluminum to produce compounds with aversive tastes on the platter surface.

Each animal was observed in each test. Latency (time to contact the first pellet in each new meal segment) and duration (time from the first contact to the swallowing of the fifth pellet) scores were recorded by hand-operated electronic timers. If all five pellets were not eaten at the end of a 36-sec period, an arbitrary "ceiling" score of $36 \mathrm{sec}$ was assigned; this conservative criterion systematically underestimated the suppressive effects of the drug on the duration scores. The number of uneaten pellets and incidents of grooming, locomotion, or freezing were noted for each trial.

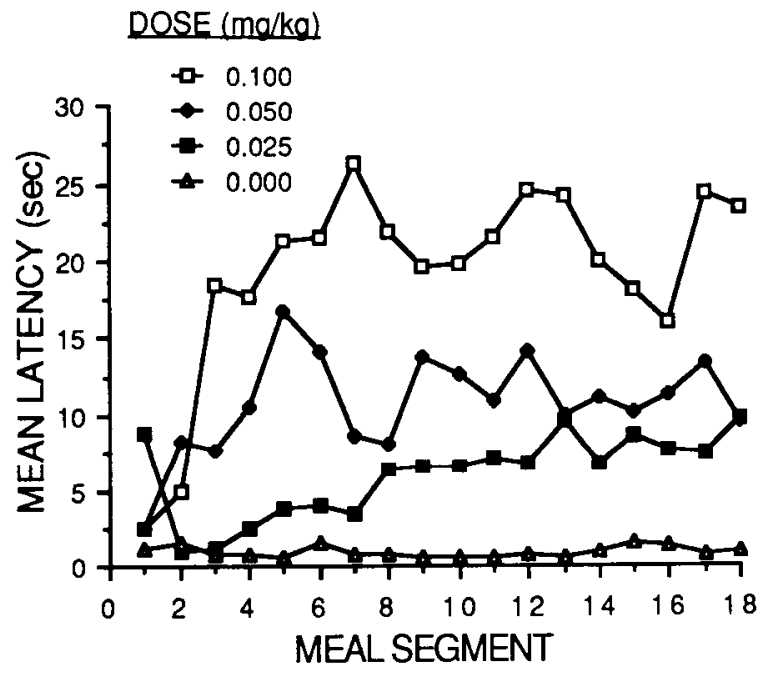

Figure 1. Response latencies under saline and three doses of SCH 23390.

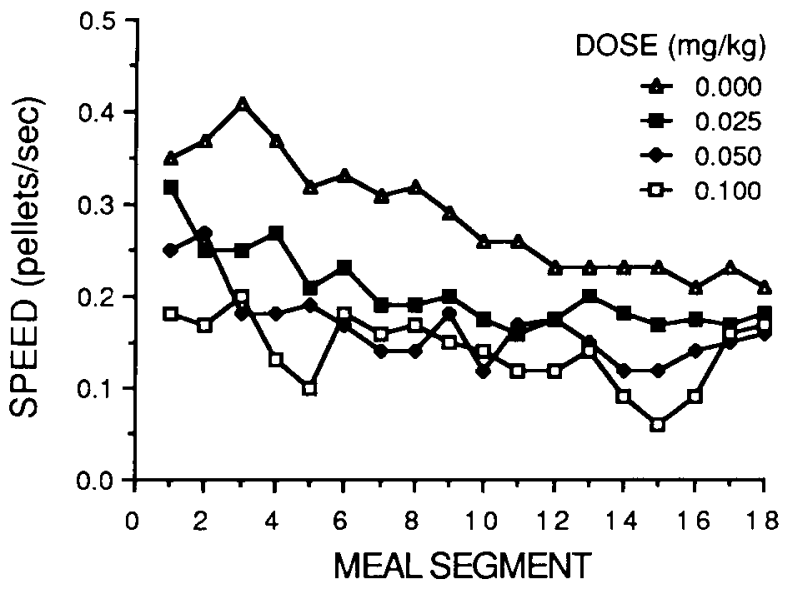

Figure 2. Eating speed under saline and SCH 23390.

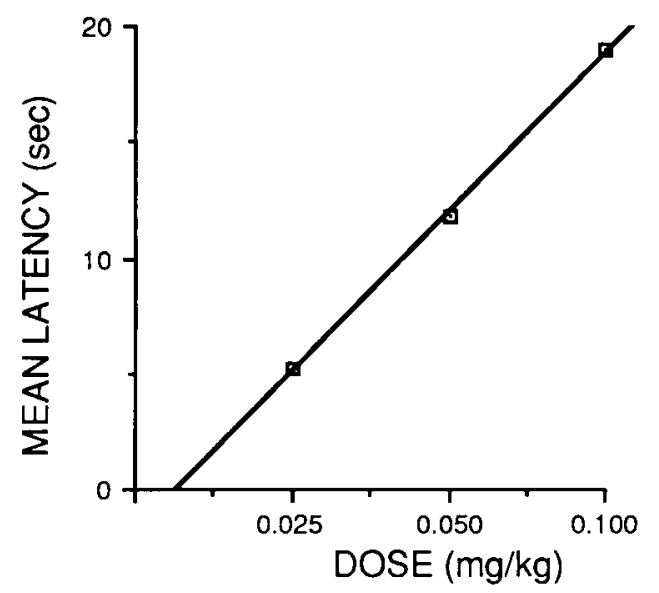

Figure 3. Mean response latency under saline and SCH 23390.

Each animal was tested under each of three doses $(0.025,0.05$, and $0.1 \mathrm{mg} / \mathrm{kg}$, calculated as the base) of the selective D-1 receptor blocker SCH 23390 , dissolved in $0.3 \%$ tartaric acid and injected in a volume of $1 \mathrm{ml} / \mathrm{kg}$. The three doses and placebo (tartaric acid) were tested in counterbalanced order every third day; drug-free testing intervened between the treatment days. The testing began $30 \mathrm{~min}$ after injection.

\section{RESULTS}

The SCH 23390 increased the eating latency; the effect developed rapidly, but progressively, over trials (Figure 1). An analysis of variance suggested that these observations were statistically reliable $[F(8,88)=32.02$, $p<.001]$. The drug also decreased the eating speed, and in this case the effect was evident from the very first trial (Figure 2); an analysis of variance again indicated statistical reliability $[F(11,121)=17.80, p<.001]$. The effects were dose-orderly (Figure 3), as were the effects of SCH 23390 on the number of pellets eaten (Figure 4).

The progressive nature of the deficits depended on the dose of SCH 23390. With the lowest dose of SCH 23390, the mean latency was normal or near normal in the early trials (with the anomalous exception of the very first trial, 


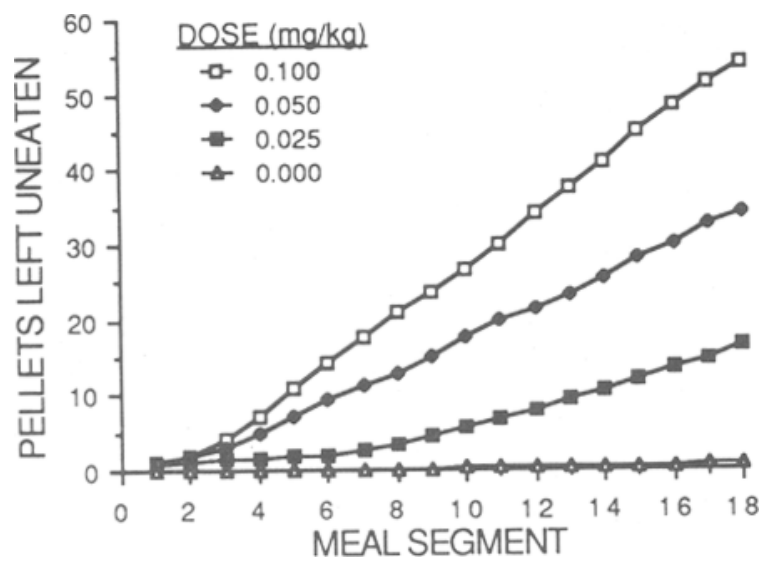

Figure 4. Pellets left uneaten under saline and SCH 23390.

which was not similarly affected by the higher doses) but lengthened progressively as the session progressed (Figure 1); the mean eating speed was slow from the very first trial; it became slower at the end of the session than it had been at the beginning (Figure 2). Pellets were left uneaten by some animals as early as the second meal segment, and all the animals were reliably leaving pellets uneaten by the seventh meal segment (Figure 4). With the middle dose, the mean latency was nearly normal on the first trial, but it increased quickly as the session progressed (Figure 1). The mean speed was slow from the first trial and became slower as the testing continued (Figure 2). Pellets were left uneaten by some animals even on the first trial, and all animals were leaving some pellets uneaten by the third or fourth trial (Figure 4). With the highest dose, the mean latency was near normal on the first two trials; but it was very long by the third trial, and it did not change further (Figure 1). The mean eating speed was slow from the first trial, and it decreased somewhat further during the session (Figure 2). Pellets were left uneaten on the first and second trials, and by the third trial the animals were leaving the same average number of pellets uneaten on each trial (Figure 4).

The mean latency was inflated by an increase in the variance of the latency scores rather than by a simple shift to the right of the distribution of scores (Figure 5). The best latencies were just under $0.3 \mathrm{sec}$; such latencies were infrequent in any case, but they were seen in the highand medium-dose conditions as well as in the control condition (Figure 5). Modal latency scores were between 0.3 and $0.5 \mathrm{sec}$ under the control conditions and also under each drug dose; it was the frequency of such scores-not their absolute value-that was decreased in a dose-orderly way by SCH 23390 . The frequency of extremely long latency scores (four times the modal latency or longer) was increased in a dose-orderly way by the drug; since food was removed after $36 \mathrm{sec}$, and since the animals had often not touched the first pellet within this time, it was not possible to identify what the worst latency would have been under drug conditions. A $t$ test for homogeneity of variance revealed that variances from the drug tests $(0.025$, 0.05 , and $0.1 \mathrm{mg} / \mathrm{kg}$ ) were significantly greater than the variance of the scores from the control test $[t s(214)=$ $-13.4,-9.4$, and -7.9 , respectively; $p<.001$ in each case]. This failure to meet the assumption of homogeneity of variance means that the analysis of variance mentioned earlier cannot be interpreted as a simple shift in the mean of a population of normally distributed scores.

The mean duration score was also inflated by an increase in the variability of eating $[t \mathrm{~s}(214)=-6.1,-6.2$, $-5.96 ; p<.001$ in each case]. Thus, again, the effect of SCH 23390 was not to shift the distribution of scores but, rather, to increase the variance by increasing extremely slow scores in the drug condition. Indeed, the drug made the distribution of duration scores bimodal (thus violating a second assumption of analysis of variance as a test of the differences between means). The most extreme scores were often associated with episodes of locomotion or grooming (see below); thus they reflected periods of other activity, not inactivity. The shortest times

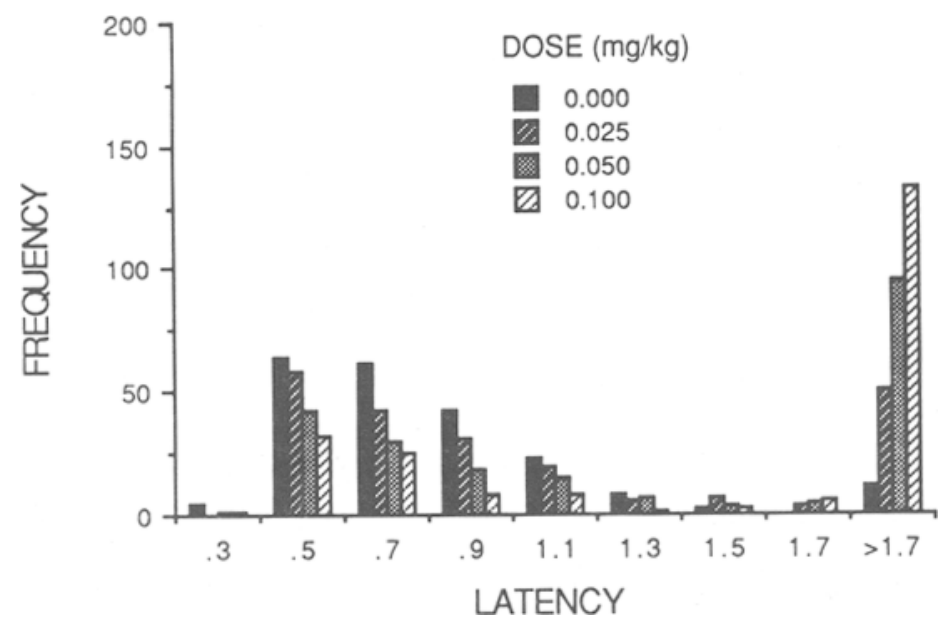

Figure 5. Relative frequencies of short and long response latencies under saline and SCH 23390. 


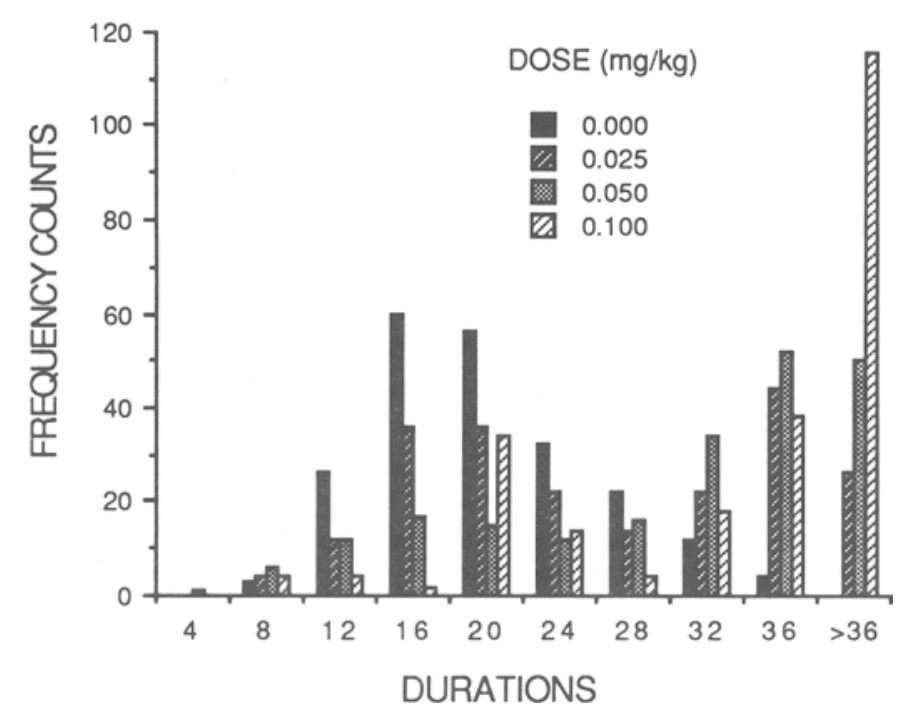

Figure 6. Relative frequencies of short and long meal segment durations under saline and SCH 23390.

to complete the consumption of all five pellets were between 4 and $8 \mathrm{sec}$ for control, high-dose, and low-dose conditions and under $4 \mathrm{sec}$ for the middle-dose condition (Figure 6). The modal peak in the control condition was at 12-16 sec; the distribution of duration scores in the drug conditions had initial peaks at $12-16 \mathrm{sec}$ for the low and middle doses and at 16-20 sec for the high dose. The distributions of the low- and middle-dose scores had a second peak at 32-36 sec, with all scores greater than $36 \mathrm{sec}$ accounting for a lower count than the scores between 32 and $36 \mathrm{sec}$. Thus, this peak was clearly a second mode for the low- and middle-dose conditions. The distribution of the high-dose scores may have peaked beyond the 36sec cutoff score; scores longer than $36 \mathrm{sec}$ were more frequent than scores between 32 and $36 \mathrm{sec}$. Whether the second mode was between 32 and $36 \mathrm{sec}$ or between 36 and $40 \mathrm{sec}$, it is clear that the distribution was again bimodal.

Observations of freezing were rare; they were seen in the saline as well as in drug conditions, and they were restricted to the first day of drug testing. Animals that did not finish their pellets by the end of a trial had usually interrupted eating to groom or explore. The first trial in which an animal failed to initiate feeding was always preceded by segments in which it initiated feeding normally, but did not complete it, and a development of reliably abnormal latency scores always followed clearly abnormal duration scores.

\section{DISCUSSION}

These data fit well with those of other studies, which suggest that a selective blockade of the D-1 dopamine receptor disrupts feeding (Schneider et al., 1986a, 1986b) and food-rewarded leverpressing (Beninger et al., 1987; Nakajima, 1986). The disruption was not a simple alter- ation of the absolute performance capacity of the animals: the absolute value of best scores was generally unaffected. As is the case with the preferential D-2 antagonist pimozide, SCH 23390 increased the number of trials in which peak performance was not seen, rather than simply adding a constant to each value in the distribution of response scores. Shifts in mean performance that result from increased variance and increased frequency of trials in which performance was poor (rather than a simple point-by-point shift of the distribution) have been offered as an operational-as well as an intuitive-definition of what we mean by a motivational deficit (Wise \& Colle, 1984; Wise \& Raptis, 1986). If a constant motoric impairment were superimposed on an animal with normal motivation, a shift in the distribution with no change in variance would be expected; this, the basic assumption of analysis of variance, is the reason why a homogeneity of variance must be assumed if the analysis of variance is to be used as a test in the differences between sample means (Scheffé, 1959).

A second finding that supports a motivational interpretation of both the present data and the effects of the D-2 antagonist pimozide (Wise \& Colle, 1984; Wise \& Raptis, 1986) is the finding that response-initiation scores were reasonably normal until the animals had gained some experience with the food under the drug condition. The capacity of food to maintain feeding at a normal pace was altered before any change in its capacity to initiate feeding. This is consistent with the notion that it is the impact of the response-maintaining, proximal cues of the food pellet (taste, smell?), rather than its response-eliciting distal cues (smell?, sight), that are most severely affected by both the D-1 and the D-2 antagonists. While both latency and duration deficits developed much more rapidly under SCH 23390 than under pimozide (Wise \& Colle, 1984; Wise \& Raptis, 1986), the progressive nature of 
the deficits and the fact that the duration deficit preceded the latency deficit reflect qualitatively similar effects of D-1 and D-2 antagonism.

The current thinking about the roles of D-1 and D-2 receptors in dopamine function revolves around the notion that the two receptors interact, such that the coactivation of the D-1 receptor is necessary for any expression of the behavioral effects of activation of the D-2 receptor (Barone, Davis, Braun, \& Chase, 1986; Carlson, Bergstrom, \& Walters, 1987; Clark \& White, 1987; Longoni, Spina, \& Di Chiara, 1987; Weick \& Walters, 1987). Thus, selective D-1 antagonists can decrease the behavioral effectiveness of selective D-2 agonists (Barone et al., 1986; Hyttel, 1983; Woolverton, 1986). The present data are consistent with this view, since selective D-1 blockade produces the same sort of behavioral effect as is caused by preferential D-2 blockade. This leaves the question, however, of why, if D-1 and D-2 blockers interfere with the function of a common dopaminergic mechanism, SCH 23390 should have the greater potency as reflected in the more rapid onset of progressive deficits in mean score.

A possible explanation for the greater persistency in responding under pimozide is that D-2 antagonists stimulate compensatory feedback effects in the dopamine system, whereas SCH 23390 does not (Clark \& White, 1987; Hand, Kasser, \& Wang, 1987). D-2 antagonists cause feedback-mediated increases in cell firing, whereas SCH 23390 does not (Hand et al., 1987). Thus the dopamine system itself, through its feedback mechanisms, may produce more resistance to the postsynaptic effects of pimozide than to those of SCH 23390: since pimozide stimulates dopamine cell firing, it leads to increased dopamine concentrations in the synapse, where dopamine and its antagonists compete for receptor sites. Since SCH 23390 does not stimulate dopamine cell firing, it does not counteract its own postsynaptic actions by increasing the competitive levels of synaptic dopamine.

Whatever the nature of D-1 and D-2 actions and interactions, it is clear that SCH 23390 attenuates feeding as well as a range of other behaviors previously attributed to D-2 receptor activation. Although performance is more severely and more quickly attenuated with the D-1 antagonist, the progressive nature of the deficit and the fact that normal performance is still seen in a significant portion of the trials make it clear that the deficit does not simply reflect a gross motoric debilitation in the animals. The animals are capable of initiating feeding normally and of feeding at normal speed, and they demonstrate this capability frequently; thus it would appear that the D-1 dopamine receptor interacts in some way with D-2 receptors that have previously been linked (Wise \& Colle, 1984; Wise \& Raptis, 1986) to motivational aspects of feeding.

\section{REFERENCES}

AHLENIUS, S. (1979). An analysis of behavioral effects produced by drug-induced changes of dopaminergic transmission in the brain. Scandinavian Joumal of Psychology, 20, 59-64.
Barbeau, A. (1974). Drugs affecting movement disorders. Annual Review of Pharmacology, 14, 91-113.

Barone, P., Davis, T. A., Braun, A. R., a Chase, T. N. (1986). Dopaminergic mechanisms and motor function: Characterization of D-1 and D-2 dopamine receptor interactions. European Joumal of Pharmacology, 123, 109-114

Beninger, R. J. Cheng, M., Hahn, B. L., Hofman, D. C., MazurSKi, E. J., MorenCy, M. A., RAmm, P., Stewart, R. J. (1987). Effects of extinction, pimozide, SCH 23390. and metoclopramide on food-rewarded operant responding of rats. Psychopharmacology. 92. 343-349.

Beninger, R. J., Freedman, N. L. (1982). The use of two operants to examine the nature of pimozide-induced decrease in responding for brain stimulation. Physiological Psychology, 10, 409-412.

Blundell, J. E., \& LATham, C. J. (1978). Pharmacological manipulation of feeding: Possible influences of serotonin and dopamine on food intake. In R. Samanin, \& S. Garattini (Eds.), Central mechan isms of anorectic drugs (pp. 83-109). New York: Raven Press.

BLUNdell, J. E., LAThaM, C. J. (1982). Behavioural pharmacology of feeding. In T. Silverstone (Ed.), Drugs and appetite (pp. 4180). London: Academic Press.

Carlson, J. H., Bergstrom, D. A., Walters, J. R. (1987). Stimulation of both $D_{1}$ and $D_{2}$ dopamine receptors appears nocessary for full expression of postsynaptic effects of dopamine agonists: A neurophysiological study. Brain Research, 400, 205-218.

Clark, D., * WhITE, F. J. (1987). Review: DI dopamine receptorthe search for a function: A critical evaluation of the D1/D2 dopamine receptor classification and its function implications. Symapse, 1, 347-388.

DE WIT, H., \& WISE, R. A. (1977). Blockade of cocaine reinforcement in rats with the dopamine receptor blocker pimozide but not with the noradrenergic blockers phentolamine or phenoxybenzamine. Canadian Journal of Psychology, 31, 195-203.

EtTEnberG, A., CAMP, C. H. (1986a). Haloperidol induces a partial reinforcement extinction effect in rats: Implications for a dopamine involvement in food reward. Pharmacology, Biochemistry \& Behavior, 25, 813-821.

EtTEnberG, A., \& CAMP, C. H. (1986b). A partial reinforcement extinction effect in water-reinforced rats intermittently treated with haloperidol. Pharmacology, Biochemistry \& Behavior, 25, 1231-1235.

Fenton, H. M., \& Liebman, J. M. (1982). Self-stimulation response decrement patterns differentiate clonidine, baclofen and dopamine antagonists from drugs causing performance deficit. Pharmacology, Biochemistry \& Behavior, 17, 1207-1212.

Fouriezos, G., Hansson, P., Wise, R. A. (1978). Neurolepticinduced attenuation of brain stimulation reward in rats. Joumal of Comparative \& Physiological Psychology, 92, 661-671.

Fouriezos, G., \& WISE, R. A. (1976). Pimozide-induced extinction of intracranial self-stimulation: Response patterns rule out motor or performance deficits. Brain Research, 103, 377-380.

Frankun, K. B. J., a McCoy, S. N. (1979). Pimozide-induced extinction in rats: Stimulus control of responding rules out motor deficit. Pharmacology, Biochemistry \& Behavior, 11, 71-75.

Galustel, C. R., Bortim, M., Gomita, Y., \& Klebanoff, L. (1982). Does pimozide block the reinforcing effect of brain stimulation? Pharmacology, Biochemistry \& Behavior, 17, 769-781.

Galustel, C. R., DAvis, A. J. (1983). Affinity for the dopamine D2 receptor predicts neuroleptic potency in blocking the reinforcing effect of MFB stimulation. Pharmacology, Biochemistry \& Behavior, 19, 867-872.

HAND, T. H., KASSER, R. J., \&ANG, R. Y. (1987). Effects of acute thioridazine, metoclopramide and $\mathrm{SCH} 23390$ on the basal activity of A9 and A10 dopamine cells. European Journal of Pharmacology, 137, 251-255.

HefFiner, T. G., Zigmond, M. J., \& Stricker, E. M. (1977). Effects of dopaminergic agonists and antagonists on feeding in intact and 6hydroxydopamine-treated rats. Joumal of Pharmacology \& Experimental Therapeutics, 201, 386-399.

HORNYKIEWICZ, O. (1975). Parkinsonism induced by dopaminergic antagonists. In D. B. Kaine, T. N. Chase, \& A. Barbeau (Eds.), Advances in neurology (pp. 155-164). New York: Raven Press. 
HytTEL, J. (1983). SCH 23390-the first selective dopamine D-1 antagonist. European Journal of Pharmacology, 91, 153-154.

LiEBmAN, J. M. (1983). Discriminating between reward and performance: A critical review of intracranial self-stimulation methodology. Neuroscience \& Biobehavioral Reviews, 7, 45-72.

Longoni, R., SPina, L., Di Chiara, G. (1987). Permissive role of D-1 receptor stimulation for the expression of D-2 mediated behavioral responses: A quantitative phenomenological study in rats. Life Sciences, 41, $2135-2145$.

LYNCH, M. R. WISE, R. A. (1985). Relative effectiveness of pimozide, haloperidol and trifluoperazine on self-stimulation rateintensity functions. Pharmacology, Biochemistry \& Behavior, 23, 777-780.

Mason, S. T., Beninger, R. J., Fibiger, H. C., Phillips, A. G. (1980). Pimozide-induced suppression of responding: Evidence against a block of food reward. Pharmacology, Biochemistry \& Behavior, 12, 917-923.

NAKAJIMA, S. (1986). Suppression of operant responding in the rat by dopamine D1 receptor blockade with SCH 23390. Physiological Psychology, 14, 111-114.

NaKajima, S., MCKenzie, G. M. (1986). Reduction of the rewarding effect of brain stimulation by blockade of dopamine D1 receptor with SCH 23390. Ppharmacology, Biochemistry \& Behavior, 24, 919-923.

RISNER, M. E., JoNES, B. E. (1976). Role of noradrenergic and dopaminergic processes in amphetamine self-administration. Pharmacology, Biochemistry \& Behavior, 5, 477-482.

RiSNER, M. E., JONES, B. E. (1980). Intravenous self-administration of cocaine and norcocaine by dogs. Psychopharmacology, 71, 83-89.

Rolls, E. T., Rolls, B. J., Kelley, P. H., Shaw, S. G., Wood, R. J., DALE, R. (1974). The relative attenuation of self-stimulation, eating and drinking produced by dopamine-receptor blockade. Psychopharmacology (Berlin), 38, 219-230.

SANGER, D. J. (1986). Response decrement patterns after neuroleptic and non-neuroleptic drugs. Psychopharmacology, 89, 98-104.

SCHEFFE, H. (1959). The analysis of variance. New York: Wiley.

Schneider, L. H., Gibis, J., \& SMITH, G. P. (1986a). D-2 selective receptor antagonists suppress sucrose sham feeding in the rat. Brain Research Bulletin, 17, 605-611.

Schneider, L. H., Gibrs, J., a Smith, G. P. (1986b). Selective D-1 or D-2 receptor antagonists inhibit sucrose sham feeding in rats. Appetite, 7, 294-295.
Spyraki, C., Fibiger, H. C., \& Phillips, A. G. (1982). Attenuation by haloperidol of place preference conditioning using food reinforcement. Psychopharmacology, 77, 379-382.

WEICK, B. G., WALTERS, J. R. (1987). Effects of $D_{1}$ and $D_{2}$ dopamine receptor stimulation on the activity of substantia nigra pars reticulata neurons in 6-hyroxydopamine lesioned rats: $D_{1} / D_{2}$ coactivation induces potentiated responses. Brain Research, 405, 234-246.

WISE, R. A. (1982). Neuroleptics and operant behavior: The anhedonia hypothesis. Behavioral \& Brain Sciences, 5, 39-87.

WISE, R. A. (1987). Sensorimotor modulation and the variable action pattern (VAP): Toward a noncircular definition of drive and motivation. Psychobiology, 15, 7-20.

Wise, R. A., Colle, L. (1984). Pimozide attenuates free feeding: "Best scores" analysis reveals a motivational deficit. Psychophar. macologia, 84, 446-451.

WISE, R. A., RAPTIS, L. (1986). Effects of naloxone and pimozide on initiation and maintenance measures of free feeding. Brain Research, 368, 62-68.

Wise, R. A., SPIndler, J., DE WIT, H., \& Gerber, G. J. (1978). Neuroleptic-induced "anhedonia" in rats: Pimozide blocks the reward quality of food. Science, 201, 262-264.

Wise, R. A., SPInDler, J., Legault, L. (1978). Major attenuation of food reward with performance sparing doses of pimozide in the rat. Canadian Journal of Psychology, 32, 77-85.

Woolverton, W. L. (1986). Effects of a $D_{1}$ and a $D_{2}$ dopamine antagonist on the self-administration of cocaine and piribedil by rhesus monkeys. Pharmacology, Biochemistry \& Behavior, 24, 531-535.

YOKEL, R. A., WISE, R. A. (1975). Increased lever pressing for amphetamine after pimozide in rats: Implications for a dopamine theory of reward. Science, 187, 547-549.

YOKEL, R. A., WISE, R. A. (1976). Attenuation of intravenous amphetamine reinforcement by central dopamine blockade in rats. Psychopharmacology, 48, 311-318.

Zarevics, P., \& Setler, P. (1979). Simultaneous rate-independent and rate-dependent assessment of intracranial self-stimulation: Evidence for the direct involvement of doparnine in brain reinforcement mechanisms. Brain Research, 169, 499-512.

(Manuscript received December 29, 1987; revision accepted for publication July 5, 1988.) 